\title{
Free Vibration Modeling and Computation of Reinforced Thermoplastic Pipe (RTP) Conveying Fluid in Off/Inshore Industry Applications
}

\author{
Lingyun $\mathrm{Xu}^{1}$, Laith K. Abbas ${ }^{1}$, Dongyang $\mathrm{Chen}^{1}$ and Xiaoting Rui ${ }^{1}$ \\ ${ }^{1}$ Institute of Launch Dynamics, Nanjing University of Science and Technology, Nanjing 210094, Jiangsu Province, P. R. China
}

\begin{abstract}
Reinforced Thermoplastic Pipe (RTP) is a composite material flexible pipe. Due to RTP superior behavior, it encouraged the industry for using to offshore and inshore applications. Marine risers and cross-country pipelines resting on elastic medium are slender flexible structures conveying fluid and exhibit complex dynamic behaviors. Therefore, it requires accurate dynamic modeling for prediction the vibration characteristics. Transfer Matrix Method for Multibody Systems (MSTMM) is one of the sophisticated methods that can be used efficiently to model large systems and calculate its vibration characteristics and dynamic responses. The size of matrices in MSTMM remains small regardless of the number of elements in the model. Having smaller matrix sizes helps to have less computational expense leading to a faster answer. Based on the MSTMM advantages and Euler-Bernoulli linear theory, transfer matrix of the pipe in both offshore and inshore industry applications is built-up for vibration analysis. Recursive eigenvalue search algorithm is used to determine the system frequencies. Numerical examples are performed to validate with those published in the open literature.
\end{abstract}

\section{Introduction}

Vibration tends to have plenty of advantages and a lot more disadvantages, stirring up engineers worldwide to put a lot effort to use its advantages and curtail it disadvantages. Invariably, everything vibrates in the world; some vibrations are good and useful; some are really negligible; some are tolerable; some annoying and the rest fall under the dangerous category. Pipes conveying fluid have become one of the important structures widely employed in industrial applications, such as oil and hydraulic pipelines, nuclear reactor cooler channels, heat exchanger, ocean mining, drug delivery, discharge lines, marine's risers, suction and pressure pipelines, fuel feeding lines in aerospace, liquid-fuel rocket piping, micro-fluidic and nano-fluidic devices. A text by Païdoussis [1] provides an excellent review of the several developments made in this field. The subject of vibration of thin pipes conveying flowing fluid is of a considerable practical interest. In such applications, most fundamental of which is to accurately compute the natural frequency. The first observation of the dynamic behavior of a fluid conveying pipe started in 1950 by Ashley and Haviland [2], when examining the aboveground TransArabian oil pipeline.

In practice, long, cross-country pipelines rest on an elastic foundation form the basis of a number of soil structure interaction problems and have many applications in geotechnical engineering. As a result, numerous research reports involving the calculation and analysis approach for pipelines on elastic foundation have been presented. Soil media may represent by some popular models, such as Winkler's model (called one-parameter foundation or simple linear model) [3-5], in which soil is represented by a series of constant stiffness (represent the compressive resistance of soil), closely spaced linear springs, is a very popular model of the soil employed in many studies, although leads to the discontinuity of the soil which was proved to be absent of enough accuracy. However, the real soil medium is more complex in its elastic behavior, where the mechanical resistance in soil arises from both the compressive and shear strains, and hence, it is more realistic to consider shear interactions between the soil springs. The Pasternak model [6] is one of the various two-parameter foundation models (for more, generalized foundation [7] and Vlasov foundation [8]), which may capture the real behavior state of the soil more precisely. Here in, between the Winkler springs and the pipe surface, an incompressible shear layer is introduced to resist only transverse shear. The springs are connected to this shear layer.

Reinforced Thermoplastic Pipe (RTP) is a kind of composite material flexible pipe, which consists of a thermoplastic liner, two-layer reinforcement and a thermoplastic protective outer covering [9]. For its outstanding behavior in eroded environment and fatigue loading (see Fig. 1), RTP is originally developed for onshore oil and gas production; however, it is now increasingly being used in selected offshore projects for the merit of corrosion and erosion resistance, light weight, reduction in life-cycle cost, high-energy efficiency, high stiffness of the laminated pipe wall (leading to excellent 
mechanical behavior in flow-induced instability conditions), high resistance to environmental condition and hazard, easy and low cost installation and operation [10]. It is already being used for offshore applications in water depths ranging from about 30 meters to 900 meters. Since the fluid-flow causes compressive axial forces, high values of the bending stiffness increase the critical values of flow velocity and improve the structural integrity of the pipeline [11]

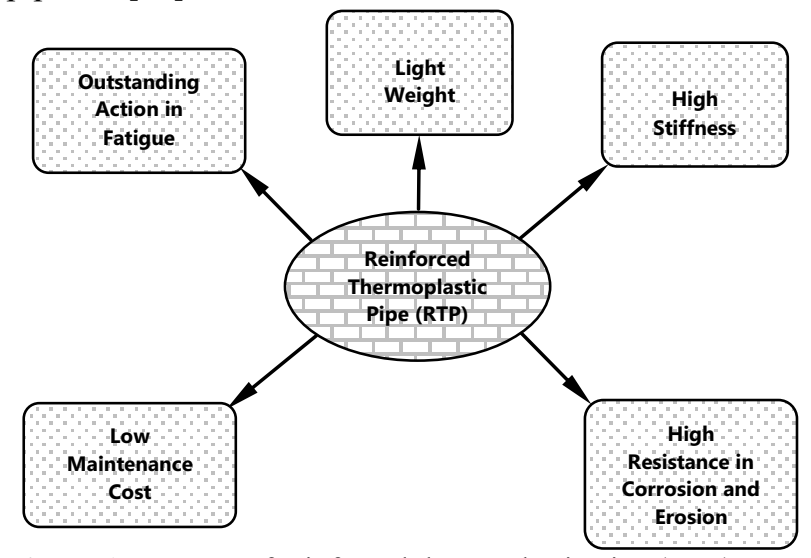

Figure 1. Features of reinforced thermoplastic pipe (RTP)

The vibration characteristics represented by the deduction of the equations of motion of pipe conveying fluid treated as Euler-Bernoulli or Timoshenko beams or even cylindrical shell for off/inshore applications using various methods (such as (1) dynamic equilibrium approach, (2) variational method, and (3) integral equation formulation) can be found in the open literature. Xiaoting Rui and colleagues [12-14] transferred the concept of State Vectors (SVs) into the classical Transfer Matrix Method (TMM), and built up a new multibody dynamics method called Transfer Matrix Method for Multibody Systems (MSTMM) or Rui Method. It is a novel and efficient method in which the order of the overall transfer matrix of the entire system is low and therefore, the computation speed is much faster than regular methods such as Finite Element Method (FEM).

In the view of MSTMM, vibrations are described in physical coordinates by kinematics (displacements/ rotations) and kinetics (internal forces/ moments) quantities, called state variables. These are small displacements $u, v, w$ along the Cartesian axes $x, y, z$ and small angular rotations $\theta_{x}, \theta_{y}, \theta_{z}$ about these axes; forces and moments are given by $q_{x}, q_{y}, q_{z}$ and $m_{x}, m_{y}, m_{z}$, respectively. In case of $3 \mathrm{D}$ modeling, the number of state variables equals $n_{s}=12$ and the physical/modal states at a connection point (between Input end $I$ and Output end $O$ of two elements) may be summarized in a column vector called State Vector (SV) denoting the mechanics state of this point [12]:

$$
\begin{aligned}
& \left.\boldsymbol{z}\right|_{\text {physical coord. }}=\left[u, v, w, \theta_{x}, \theta_{y}, \theta_{z}, m_{x}, m_{y}, m_{z}, q_{x}, q_{y}, q_{z}\right]^{T} \\
& \left.\boldsymbol{Z}\right|_{\text {modal coord. }}=\left[U, V, W, \Theta_{x}, \Theta_{y}, \Theta_{z}, M_{x}, M_{y}, M_{z}, Q_{x}, Q_{y}, Q_{z}\right]^{T}
\end{aligned}
$$

Details about MSTMM theorem and strategy are given in [13-15] for the interested readers.
In the present paper, under the view of MSTMM methodology, the governing differential equations of motion of two reinforced thermoplastic pipe industry applications (Offshore and Inshore) which leading to exact free vibration frequencies are formulated. In both applications, RTP is treated as Euler-Bernoulli beam. In case of offshore application, a vertical pipe (which may call riser) is used to carry oil from a hole (well) under the sea bottom to a floating facility. The pipe is top tensioned and conveying fluid. While for the inshore industry application, fluid-conveying pipe surrounded by a viscoelastic (soil) medium has been considered. Then, the governing differential equations of the transverse vibration are transformed to an algebraic transfer equation. According to the MSTMM topology (such as chain or tree [15]) of the mulitbody system, the element transfer matrices are assembled. After eliminating the boundary conditions, a system of linear algebraic equations is established by a so-called overall transfer equation, where the coefficient matrix needs to be singular (requiring its determinant $\Delta$ to be zero). To this end, a recursive scanning approach [16] for minima of $|\Delta|$ is applied for finding the vibration frequencies. To demonstrate the accuracy, numerical examples are performed and compared with those published in the open literature.

\section{Theory}

\subsection{Offshore Industry Application: Equation of Motion and the Solution}

One of the most critical components concerning offshore industry is the riser, which connects the floating platform with the wellhead at the seabed (see Fig. 2(a)). The riser is used for carrying oil, drilling and environmental protection, and it is subjected to large forces, due to waves and underwater currents. To analyze this system, a rectangular Cartesian coordinate system oxyz is defined in a way that its origin is located at the seabed, and the $x$-axis coincides with the riser in its unreformed state. The pipe has dimensions given by the length $(L)$, the cross-sectional outer diameter $(D)$ and the thickness $(t)$ as sketched in Fig. 2(b) under the view of MSTMM. It is assumed that the pipe is sufficiently slender, that is $(D / L<<0.1)$. The motion of the riser is modelled by a tensioned linear beam (the axial tension in the riser is a linear function of depth caused by its submerged dead weight) and its lateral motion $v$ to be small as compared to the diameter of the pipe. Therefore, in the present paper, a riser conveying a fluid is considered as an EulerBernoulli beam, and it is assumed that the riser moves only in the plane oxy. One end of the pipe is clamped (rigidly connected to the sea bottom), whereas the other end of the pipe is pinned (flexibly connected to the floating platform by torsional spring with stiffness $K_{z}^{\prime}$ ). By this connection, both sides of the pipe are prevented from lateral displacement; however, it provides a restoring moment proportional to the rotation angle of the pipe. This paper adopts the differential equations in [17]. The equation of motion for forced vibration governing the 
transverse (in-plane) motion of pipe conveying fluid under top tension and hydrodynamic forces based on Morison approach as a function of axial distance $x$ and time $t$ maybe written in the following form:
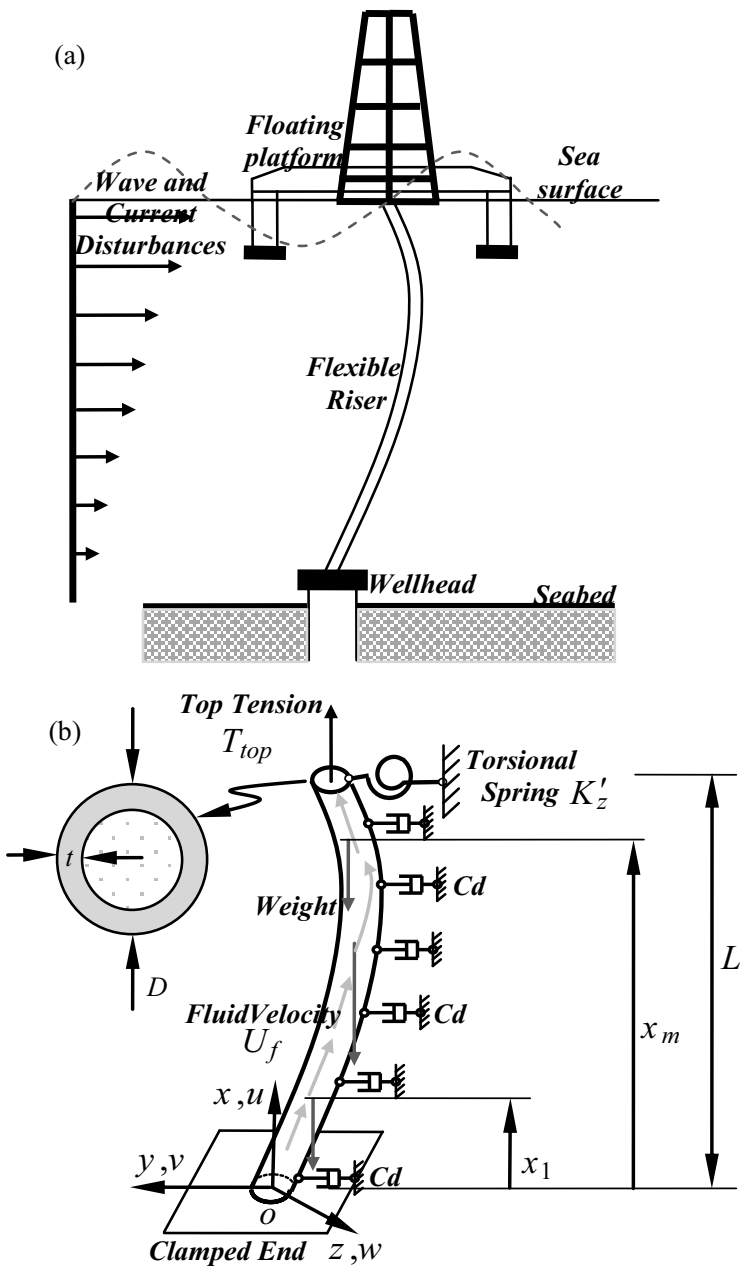

Figure 2. (a) Principle sketch of a marine riser system, and (b) Clamped-pinned pipe conveying fluid under top tension in the view of MSTMM.

$$
E I_{z} \frac{\partial^{4} v}{\partial x^{4}}-T \frac{\partial^{2} v}{\partial x^{2}}+m_{r} \frac{\partial^{2} v}{\partial t^{2}}=f_{\text {int }}+f_{\text {ext }}
$$

where

$$
\begin{aligned}
& T_{e f f}=T+\left.\left[W x+A_{e} P_{e}-A_{i} P_{i}\right]\right|_{j}= \\
& T+\left.\left[W x+A_{e} \gamma_{w}(L-x)-A_{i} \gamma_{f}(L-x)\right]\right|_{j}= \\
& \sum_{j=1}^{m}\left[W_{j} x_{j}+A_{e} \gamma_{w}\left(L-x_{j}\right)-A_{i} \gamma_{f}\left(L-x_{j}\right)\right] ; \\
& f_{\text {int }}=-\left.m_{f} \frac{d^{2} v}{d t^{2}}\right|_{x=U_{f} t}=-m_{f}\left\{\left.\frac{d}{d t}\left(\frac{\partial v}{\partial t}+\frac{\partial v}{\partial x} \frac{d x}{d t}\right)\right|_{x=U_{f} t}\right\}= \\
& -m_{f}\left\{\left.\frac{d}{d t}\left(\frac{\partial v}{\partial t}+U_{f} \frac{d v}{d x}\right)\right|_{x=U_{f} t}\right\}= \\
& -m_{f}\left\{\frac{\partial^{2} v}{\partial t^{2}}+2 U_{f} \frac{\partial^{2} v}{\partial x \partial t}+U_{f}^{2} \frac{\partial^{2} v}{\partial x^{2}}\right\} ; \\
& f_{e x t}=-\rho_{w} A_{e} C_{a} \frac{\partial^{2} v}{\partial t^{2}}-\frac{1}{2} \rho_{w} D U_{e x t} C_{d} \frac{\partial v}{\partial t}
\end{aligned}
$$

In Eq. (2) above, $E$ is the modulus of elasticity of the pipe material, $I$ is the second moment of inertia of the pipe, $m_{r}$ is the pipe mass per unit length, $T$ and $T_{\text {eff }}$ are the axial and effective tensions, respectively, $f_{\text {int }}$ and $f_{\text {ext }}$ are the internal and external forces acting on the pipe, respectively, $A_{i}$ and $A_{e}$ are the internal and external cross-sectional areas of the pipe, respectively, $P_{e}$ and $P_{i}$ are the hydrostatic pressures outside and inside the pipe segment $j$, respectively, $W$ is the weight per unit length of pipe segment $j, \rho_{w}$ is the seawater density, $\gamma_{w}$ and $\gamma_{f}$ are the specific weights of seawater and fluid, respectively, $m_{f}$ and $U_{f}$ are the fluid mass per unit length and fluid flow velocity, respectively, $C_{a}$ and $C_{d}$ are added mass coefficient and linearized drag coefficient, respectively. $U_{e x t}$ is the external flow velocity. Now the equation of motion for forced vibration reads:

$$
\begin{aligned}
& \underbrace{E I_{z} \frac{\partial^{4} v}{\partial x^{4}}}_{\text {Stiffness term }}+\underbrace{\left(m_{f} U_{f}^{2}-T_{e f f}\right) \frac{\partial^{2} v}{\partial x^{2}}}_{\text {Curvature term }}+\underbrace{2 m_{f} U_{f} \frac{\partial^{2} v}{\partial x \partial t}}_{\text {Coriolis force term }}+ \\
& \underbrace{\left(m_{r}+m_{f}+\rho_{w} A_{e} C_{a}\right) \frac{\partial^{2} v}{\partial t^{2}}}_{\text {Inertia force term }}+\frac{1}{2} \rho_{w} D U_{e x t} C_{d} \frac{\partial v}{\partial t}=0
\end{aligned}
$$

Define the following variables as dimensionless quantities:

$$
\begin{aligned}
& \xi=x / L ; v=v / L ; \beta=T_{e f f} L^{2} / E I_{z} ; \Gamma=U_{f} \sqrt{m_{f} / T_{e f f}} ; \\
& \alpha=L \sqrt{m_{f} T_{e f f} / m E I_{z}} ; \tau=t \sqrt{E I_{z} / m} / L^{2} ; \\
& \gamma=\rho_{w} D C_{d} U_{e x t} L^{2} / 2 \sqrt{m E I_{z}} ; \\
& m=m_{r}+m_{f}+\rho_{w} A_{e} C_{a}
\end{aligned}
$$

Substituting the above dimensionless variables into Eq. (3), one may have the following equation:

$$
\frac{\partial^{4} v}{\partial \xi^{4}}+\beta\left(\Gamma^{2}-1\right) \frac{\partial^{2} v}{\partial \xi^{2}}+2 \Gamma \alpha \frac{\partial^{2} v}{\partial \xi \partial \tau}+\frac{\partial^{2} v}{\partial \tau^{2}}+\gamma \frac{\partial v}{\partial \tau}=0
$$

Under free vibration, the transverse elastic deflection $v(\xi, \tau)$ of the riser in Eq. (5) may be expressed as:

$$
v(\xi, \tau)=\eta(\xi) e^{\lambda \tau}
$$

where $\eta(\xi)$ is the amplitude and $\lambda$ is the eigenvalue of the riser (In general, $\lambda=-\lambda^{\mathrm{Re}} \pm i \lambda^{\mathrm{Img}}$. The real part $\lambda^{\mathrm{Re}}$ is typically negative and related to the magnitude of damping, whereas the imaginary part $\lambda^{\mathrm{Img}}$ is related to the vibration frequency of the damped system. For undamped systems, $\lambda^{\text {Re }}=0$ and $\lambda^{\operatorname{Img}}=\omega / \sqrt{E I_{z} / m L^{4}}$, where $\omega$ is the dimension circular frequency (radian)). Substituting Eq. (6) into Eq. (5), one may get:

$$
\frac{\partial^{4} \eta}{\partial \xi^{4}}+\beta\left(\Gamma^{2}-1\right) \frac{\partial^{2} \eta}{\partial \xi^{2}}+2 \Gamma \alpha \lambda \frac{\partial \eta}{\partial \xi}+\left(\lambda \gamma+\lambda^{2}\right) \eta=0
$$

Equation (7) is a fourth order ordinary differential equation that can be solved using standard power series method:

$$
\eta(\xi)=\sum_{j=1}^{4} A_{j} e^{i P_{j} \xi}
$$


where $P_{j}, j=1, \mathrm{~L}, 4$ are the four roots of the fourth order polynomial (or called wave numbers) and $A_{j}$ are amplitudes of vibration. Substituting the solution of Eq. (8) into Eq. (7), one may have the characteristic equation for offshore pipe:

$$
P^{4}-\beta\left(\Gamma^{2}-1\right) P^{2}+2 \Gamma \alpha \lambda i P+\left(\lambda \gamma+\lambda^{2}\right)=0
$$

As a function of any selected values of the riser parameters $(\beta, \Gamma, \alpha, \gamma$ and $\lambda)$, the associated eigenvalues can be determined from Eq. (9) and it may have complex roots.

\subsection{Inshore Industry Application: Equation of Motion and the Solution}

There are assumptions made for the deduction of the equations governing the motion of the pipeline as follows: (1) The pipe is long and straight, thus the Euler-Bernoulli beam theory is applicable for description of the pipe dynamic bending; (2) the motions are small, therefore, in this case the system can be analyzed by the linear theory; and (3) the effects of internal pressure and external forces are neglected in the analysis. Figure 3 (a and b) illustrate schematically a uniform fluid conveying pipe resting on foundation and its corresponding mathematical model in the view of MSTMM, respectively [18].

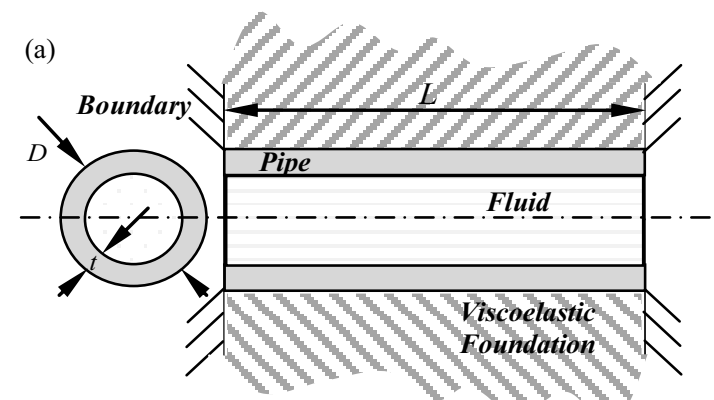

(b)

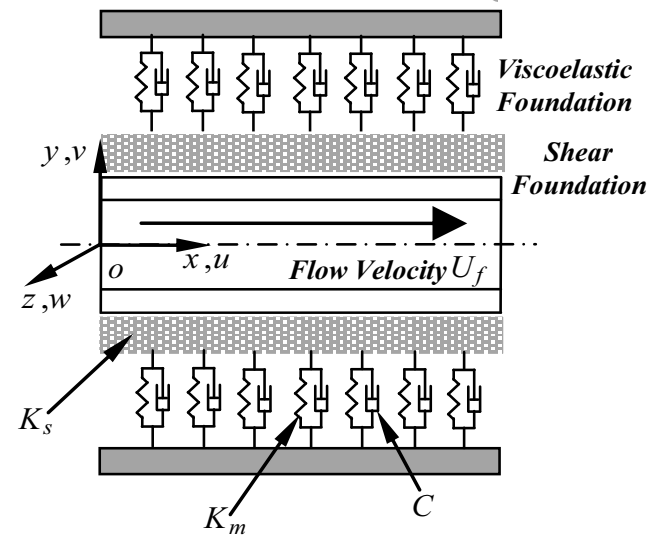

Figure 3. (a) Schematic of clamped-clamped pipe conveying fluid resting on viscoelastic foundation, and (b) Mathematical model in the view of MSTMM showing a beam resting on a foundation modeled using springs and dashpots with shear interaction.

The dynamic response of an Euler-Bernoulli beam of length $L$ with constant cross-section and lateral displacement $v(x, t)$, resting on a viscoelastic foundation represented by dashpots and springs with shear interaction and subjected to an external force is given by:

$\underbrace{E I_{z} \frac{\partial^{4} v}{\partial x^{4}}}_{\text {Elastic force }}+\underbrace{m_{f} U_{f}^{2} \frac{\partial^{2} v}{\partial x^{2}}}_{\begin{array}{c}\text { inertia force of the fluid } \\ \text { flowing in a curved path }\end{array}}+\underbrace{2 m_{f} U_{f} \frac{\partial^{2} v}{\partial x \partial t}}_{\begin{array}{c}\text { inertia force due to } \\ \text { Coriolis acceleration }\end{array}}+\underbrace{\left(m_{r}+m_{f}\right) \frac{\partial^{2} v}{\partial t^{2}}}_{\begin{array}{c}\text { inertia force due } \\ \text { to the acceleration } \\ \text { of the pipe with fluid }\end{array}}=F ;$

where $F=\underbrace{K_{S} \frac{\partial^{2} v}{\partial x^{2}}-C \frac{\partial v}{\partial t}-K_{m} v}_{\text {visocelastic foundation }} ; \quad$ Combined:

$E I_{z} \frac{\partial^{4} v}{\partial x^{4}}+\left(m_{f} U_{f}^{2}-K_{S}\right) \frac{\partial^{2} v}{\partial x^{2}}+2 m_{f} U_{f} \frac{\partial^{2} v}{\partial x \partial t}+\left(m_{r}+m_{f}\right) \frac{\partial^{2} v}{\partial t^{2}}$

$+C \frac{\partial v}{\partial t}+K_{m} v=0$

where $C$ and $K_{m}$ are the coefficient of viscous damping and Winkler's spring constant of the viscoelastic foundation, respectively. $K_{s}$ is the shear parameter of the foundation.

Using the following dimensionless quantities:

$$
\begin{aligned}
& \xi=x / L ; v=v / L ; m=m_{f} /\left(m_{f}+m_{r}\right) ; \\
& u=L U_{f} \sqrt{m_{f} / E I_{z}} ; \varepsilon=L^{2} C / \sqrt{E I_{z}\left(m_{f}+m_{r}\right)} ; \\
& \tau=t \sqrt{E I_{z} /\left(m_{f}+m_{r}\right)} / L^{2} ; \hat{k}_{m}=K_{m} L^{4} / E I_{z} ; \\
& \hat{k}_{s}=K_{s} L^{2} / E I_{z}
\end{aligned}
$$

Then, Eq. (10) with Eq. (11) may take the following form after substituting $v(\xi, \tau)=\eta(\xi) e^{\chi \tau}$, where $\chi$ is the eigenvalue of the system $\left(\chi=-\chi^{\mathrm{Re}} \pm i \chi^{\mathrm{Img}}\right.$, similarly for $\lambda$ in Section 2.1):

$$
\frac{\partial^{4} \eta}{\partial \xi^{4}}+\left(u^{2}-\hat{k}_{s}\right) \frac{\partial^{2} \eta}{\partial \xi^{2}}+2 u \chi \sqrt{m} \frac{\partial \eta}{\partial \xi}+\left(\chi \varepsilon+\chi^{2}+\hat{k}_{m}\right) \eta=0
$$

Similarly in Section 2.1, substituting the solution of Eq. (8) into Eq. (12), the following characteristic equation for inshore pipe can be obtained:

$$
P^{4}-\left(u^{2}-\hat{k}_{s}\right) P^{2}+2 i u \chi \sqrt{m} P+\left(\chi \varepsilon+\chi^{2}+\hat{k}_{m}\right)=0
$$

\subsection{Bending Stiffness of RTP}

A typical construction of RTP consisting of three layers is shown in Fig. 4. The liner and cover layers are made normally of High Density Polyethylene (HDPE), which are used to containing fluid and providing external protection, respectively. However, some are made by Cross Linked Polyethylene (PEX) or Polyamide (PA) or Polyvinylidene Fluoride (PVDF). The middle layer includes two reinforced layers with nominally $\theta= \pm 54.7^{\circ}$ helical winding angle; the reinforced tape is a combination of matrix (usually HDPE or PA or PVDF) and twisted aramid fibers. These three layers can be considered as perfect bonded because they are formed by heating extrusion during the manufacturing process [9] Due to the anisotropy of RTP materials, it is convenient to adopt a sufficient and accurate method to compute the equivalent bending stiffness as follows [11]: 


$$
R_{e q}=\frac{\pi D}{2}\left(\frac{D}{2 a_{11}}+\frac{1}{d_{11}}\right)
$$

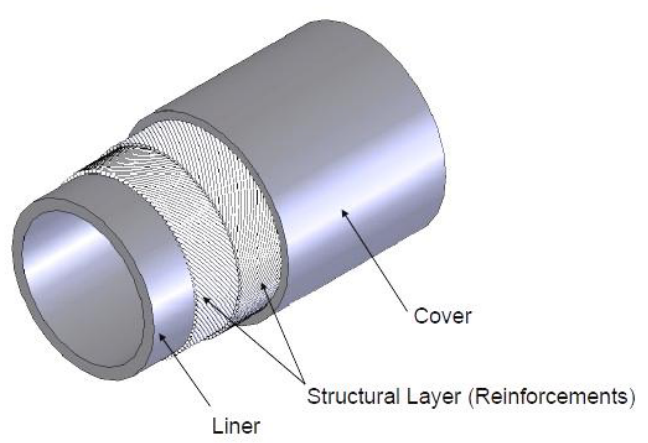

Figure 4. RTP construction

Inverted the stiffness matrix [ABD] of the RTP laminate [19] in which their parameters depend mainly on the mechanical properties $\left(E_{1}, E_{2}, G_{12}, v_{12}\right)$ and fiber orientation angle $(\theta)$ of the RTP, then the variables $a_{11}$ and $d_{11}$ in Eq. (14) can be evaluated. The bending stiffness $E I_{z}$ appears in the stiffness term of Eq. (3) (offshore pipe) and the elastic force of Eq. (10) (inshore pipe) can be now replaced by the equivalent bending stiffness $R_{e q}$ for the RTP applications.

\subsection{MSTMM: Transfer Matrix of the Pipe, Overall Transfer Equation and Characteristic Equation Solution}

By using the wave numbers $P_{j}, j=1, \mathrm{~L}, 4$ calculated from the characteristic equations of the offshore and inshore pipes given in Eqs. (9 and 13), respectively, the vibration mode of the lateral displacement of beam segment $l$ in the modal coordinates with the four unknown constants $A_{j}$ can be written as follows:

$$
V=l *\left(A_{1} e^{i P_{1} x / l}+A_{2} e^{i P_{2} x / l}+A_{3} e^{i P_{3} x / l}+A_{4} e^{i P_{4} x / l}\right)
$$

The state variables in the $\boldsymbol{Z}$ (Eq. (1)) may be condensed to the essential coordinates according to the Euler-Bernoulli beam theory vibrating in $x-y$ plane, i.e., $n_{s}=4, \boldsymbol{Z}=\left[V, \Theta_{z}, M_{z}, Q_{y}\right]^{T}$. The corresponding bending rotation $\Theta_{z}$, bending moment $M_{z}$ and shear force $Q_{y}$ may be expressed by, respectively:

$$
\Theta_{z}=\frac{\mathrm{d} V}{\mathrm{~d} x} ; \quad M_{z}=E I_{z}\left(R_{e q}\right) \frac{\mathrm{d}^{2} V}{\mathrm{~d} x^{2}} ; \quad Q_{y}=E I_{z}\left(R_{e q}\right) \frac{\mathrm{d}^{3} V}{\mathrm{~d} x^{3}}
$$

One of the MSTMM topologies used to express the system dynamic behavior is chain topology. For a general element $j$ with single input end $(I)$ and single output end $(O)$, the transfer equation (TE) can be obtained as $\boldsymbol{Z}_{O}=\boldsymbol{U}_{j} \boldsymbol{Z}_{I}$ where $\boldsymbol{U}_{j}$ is the transfer matrix of the $j^{\text {th }}$ element expressing the relationship between the modal SVs of its output end $\boldsymbol{Z}_{O}$ and its input end $\boldsymbol{Z}_{I}$. By combining Eqs. (15 and 16), one may obtain the transfer matrix $\boldsymbol{U}$ as follows:

$$
\begin{aligned}
& \boldsymbol{Z}=\boldsymbol{U} \boldsymbol{A} ; \\
& \text { where } \\
& \left.\boldsymbol{U}\right|_{4 \times 4}=\left[\begin{array}{ccc}
U_{11} & \cdots & U_{14} \\
\vdots & \ddots & \vdots \\
U_{41} & \cdots & U_{44}
\end{array}\right] ; \boldsymbol{Z}=\left[V, \Theta_{z}, M_{z}, Q_{y}\right]^{T} ; \\
& \boldsymbol{A}=\left[A_{1}, A_{2}, A_{3}, A_{4}\right]^{T}
\end{aligned}
$$

(a) (b)

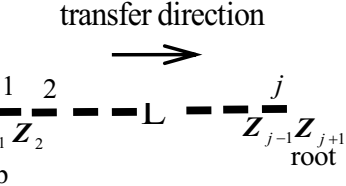

Figure 5. Clamped-clamped beam (a) and (b) corresponding state vectors and transfer direction.

The coefficient vector $\boldsymbol{A}$ of unknown constants can be adopted as boundary condition $\boldsymbol{Z}_{I}$ at the beam segment input end $(x=0)$, such as $\boldsymbol{Z}_{I}=[\boldsymbol{U}(0)] \boldsymbol{A}$ or $\boldsymbol{A}=[\boldsymbol{U}(0)]^{-1} \boldsymbol{Z}_{I}$. For the beam segment output end at ( $x=l$ ), from Eq. (17) the output condition can be found:

$$
\boldsymbol{Z}_{O}=[\boldsymbol{U}(l)] \boldsymbol{A}=[\boldsymbol{U}(l)][\boldsymbol{U}(0)]^{-1} \boldsymbol{Z}_{I}=\boldsymbol{U}^{\text {pipe }} \boldsymbol{Z}_{I}
$$

where, $\boldsymbol{U}^{\text {pipe }}$ is $4 \times 4$ element transfer matrix of the offshore/inshore pipe according to the values of $P_{j}$. For several elements as an example of clamped-clamped beam shown in Fig. 5, it can be assembled to provide the transfer matrix of the whole system according to the chain topology as follows:

$$
\begin{aligned}
& \boldsymbol{Z}_{O}=\boldsymbol{T} \boldsymbol{Z}_{I} ; \boldsymbol{T}=\prod_{k=0}^{j-1} \boldsymbol{U}_{j-k} ; \boldsymbol{U}_{\text {all }} \boldsymbol{Z}_{\text {all }}=\boldsymbol{0} ; \\
& \text { where } \\
& \boldsymbol{Z}_{\text {all }}^{T}=\left[\begin{array}{ll}
\boldsymbol{Z}_{I}^{T} & \boldsymbol{Z}_{O}^{T}
\end{array}\right]^{T} \text { and } \boldsymbol{U}_{\text {all }}=\left[\begin{array}{ll}
\boldsymbol{T} & -\boldsymbol{I}
\end{array}\right],
\end{aligned}
$$

$\boldsymbol{I}$ is identity matrix

Observing the overall transfer equation (Eq. 19), it shows that $\boldsymbol{Z}_{\text {all }}$ contains only the SVs at the boundaries (root and tips). However, the SVs at the other connection points do not appear. This is one of the MSTMM pretty features. Due to known constraints of the ordinary boundary conditions of the beam, half of the state variables of $\boldsymbol{Z}_{\text {all }}$ are zeros. As a result, Eq. (19) $\left(\boldsymbol{U}_{\text {all }} \boldsymbol{Z}_{\text {all }}=\boldsymbol{0}\right)$ is reduced to $\overline{\boldsymbol{U}}_{\text {all }} \overline{\boldsymbol{Z}}_{\text {all }}=\boldsymbol{0}$. It's clear now that $\overline{\boldsymbol{Z}}_{\text {all }}$ contains the unknown state variables at the boundaries. Eliminating all the columns of $\boldsymbol{U}_{\text {all }}$ associated zeros in $\boldsymbol{Z}_{\text {all }}$ yields a square matrix $\overline{\boldsymbol{U}}_{\text {all }}$ which is only a function of the system unknown dimensionless frequency $(\lambda$ or $\chi)$. For non-trivial solutions, the characteristic equation $\Delta(\lambda$ or $\chi)=\operatorname{det} \overline{\boldsymbol{U}}_{\text {all }} \stackrel{!}{=} 0$ has to be fulfilled. A reliable and efficient algorithm called recursive scanning approach [16] is implemented in the present work to solve the characteristic equation. 
Table 1. Numerical comparison of MSTMM with [20-21] for the first three natural frequencies parameters $\sqrt{\bar{\omega}}$ of a clamped-clamped beam resting on an elastic foundation

\begin{tabular}{|c|c|c|c|c|c|c|c|c|}
\hline \multirow{2}{*}{$\hat{k}_{m}$} & \multicolumn{5}{|c|}{$\bar{K}_{S}=0.5$} & \multicolumn{4}{|c|}{$\bar{K}_{S}=2.5$} \\
\cline { 2 - 9 } & {$[20]$} & {$[21]$} & {$[22]$} & MSTMM & {$[20]$} & {$[21]$} & {$[22]$} & MSTMM \\
\hline \multirow{2}{*}{100} & 5.23 & 5.071 & 5.0718 & 5.070679 & 5.48 & 5.477 & 5.4783 & 5.477297 \\
\cline { 2 - 9 } & 8.16 & 8.017 & 8.0169 & 8.016814 & 8.42 & 8.423 & 8.4234 & 8.423248 \\
\cline { 2 - 8 } & 11.24 & 11.104 & 11.0998 & 11.10454 & 11.44 & 11.444 & 11.4400 & 11.44458 \\
\hline \multirow{2}{*}{10000} & 10.16 & 10.137 & 10.1373 & 10.13741 & 10.41 & 10.194 & 10.1942 & 10.19428 \\
\cline { 2 - 8 } & 10.94 & 10.883 & 10.8827 & 10.88350 & 11.38 & 11.055 & 11.0539 & 11.05464 \\
\cline { 2 - 8 } & 12.68 & 12.588 & 12.5832 & 12.58759 & 13.21 & 12.825 & 12.8209 & 12.82517 \\
\hline
\end{tabular}

Table 2. Comparison of MSTMM with [23] of the fundamental frequency parameter $\chi_{1}$ for fluid conveying pipes $u=2$, two values of Winkler foundation $\hat{k}_{m}, \hat{k}_{s}=0$ and $m=0.5$.

\begin{tabular}{|c|c|c|c|c|}
\hline \multirow{2}{*}{ Boundary Condition } & \multicolumn{4}{|c|}{$\hat{k}_{m}$} \\
\cline { 2 - 5 } & \multicolumn{3}{|c|}{0.5} & \multicolumn{2}{|c|}{1000} \\
\cline { 2 - 5 } & Table 8 [23] & MSTMM & Table 8 [23] & MSTMM \\
\hline Pinned-Pinned & 7.487 & 7.48567 & 31.878 & 31.86064 \\
\hline Clamped-Pinned & 13.631 & 13.6255 & 33.962 & 33.94626 \\
\hline Clamped-Clamped & 20.964 & 20.9566 & 37.577 & 37.56392 \\
\hline
\end{tabular}

Table 3. Numerical comparison of MSTMM with [24-26] for the first five natural frequencies (rad/sec) of a pinned-pinned riser with bending and hydrostatic fluid effects.

\begin{tabular}{|c|c|c|c|c|c|}
\hline Order & $\begin{array}{c}\text { Analytical } \\
{[24]}\end{array}$ & $\begin{array}{c}\text { Numerical } \\
{[25]}\end{array}$ & $\begin{array}{c}\text { FEM } \\
60 \text { Elements [26] }\end{array}$ & $\begin{array}{c}\text { WKB-DSSM } \\
\text { 5 Elements [26] }\end{array}$ & $\begin{array}{c}\text { MSTMM } \\
\text { 2 Elements }\end{array}$ \\
\hline 1 & 0.8150 & 0.82246 & 0.8150 & 0.8150 & 0.82093014 \\
\hline 2 & 1.8036 & 1.80327 & 1.8038 & 1.8037 & 1.81422300 \\
\hline 3 & 3.0876 & 3.08190 & 3.0879 & 3.0878 & 3.09883067 \\
\hline 4 & 4.7375 & 4.72809 & 4.7377 & 4.7377 & 4.75140758 \\
\hline 5 & 6.7890 & 6.77955 & 6.7896 & 6.7896 & 6.80334589 \\
\hline
\end{tabular}

\section{Numerical Examples}

To validate the accuracy and efficiency of the proposed model based on the MSTMM methodology in the context of free vibration, numerical examples are performed concerning the inshore and offshore pipe applications.

A comparison with the results given in [20-22] is shown in Table 1 where the non-dimensional free frequency coefficients $\sqrt{\bar{\omega}}=\sqrt{\omega}\left(\left(m_{f}+m_{r}\right) L^{4} / E I_{z}\right)^{1 / 4}$ have been given as functions of the two soil parameters for clamped-clamped beam resting on an elastic foundation. It should be noted that for the sake of comparison, the definition $\bar{K}_{S}=\hat{k_{s}} \pi^{2}$ is used here.

The following data are considered:

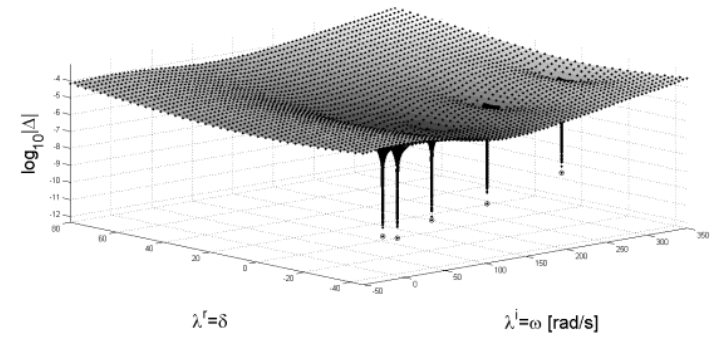

Figure 6. fMin2D function determinant of $\overline{\boldsymbol{U}}_{\text {all }}$ $\left(\hat{k}_{m}=10000, \bar{K}_{S}=2.5\right)$.

$$
\begin{aligned}
& m_{f}=0 ; U_{f}=0 ; C=0 ; L=1 \mathrm{~m} ; E I_{z}=1.266 \times 10^{3} \mathrm{~N} \cdot \mathrm{m}^{2} ; \\
& m_{r}=1.13333 \mathrm{~kg} / \mathrm{m}
\end{aligned}
$$

A single element which represented by one transfer matrix $\boldsymbol{U}^{\text {pipe }}$ has been used for the beam modeling in MSTMM. For the beam common boundary conditions, always half of the state variables are zero due to constraints, whereas the others are unknown. For clamped-clamped boundaries, we have $\boldsymbol{Z}=\left[0,0, M_{z I}, Q_{y I}, 0,0, M_{z O}, Q_{y O}\right]^{T}$. Thus, $\overline{\boldsymbol{Z}}_{\text {all }}$ in Eq. (19) will be $\overline{\boldsymbol{Z}}_{\text {all }}=\left[M_{z I}, Q_{y I}, M_{z O}, Q_{y O}\right]^{T}$ which composed of the unknown state variables only. Eigenvalue search of $\bar{U}_{\text {all }}$ is carried out with fMin2D algorithm on frequency and damping ranges are $\omega \in[-50,350] \quad \mathrm{rad} / \mathrm{sec}$, $\delta \in[-45,80] \quad, \quad N_{x 0} \times N_{y 0}=80 \times 40$ grid size, $\varepsilon_{x}=\varepsilon_{y}=10^{-4}$ absolute precision tolerance. Figure 6 shows fMin2D function determinant of the system $\left(\hat{k}_{m}=10000, \bar{K}_{S}=2.5\right)$. Table 1 shows good agreement between MSTMM and the results listed in [20-22].

Next, another comparison results with [23] obtained for the condition where $\hat{k}_{s}=0$ are presented. This represents the presence of only the Winkler foundation. Table 2 tabulates the fundamental frequency parameter $\chi_{1}$ for fluid conveying pipes $u=2$, the Winkler foundation parameter $\hat{k}_{m}, m=0.5$ and three boundary conditions. Observation on Table 2, it is shown that the 
present numerical results have an excellent agreement with [23].

An example of a uniform drilling riser under linearly varying tension is performed. The parameters of a pinnedpinned riser are [24-26]:

$L=152.4 \mathrm{~m} ; D=0.6096122 \mathrm{~m} ; t=0.015875 \mathrm{~m} ; E=2.0684189 \times 10^{11} \mathrm{~N} / \mathrm{m}^{2} ;$

$m_{r}=995.909391 \mathrm{~kg} / \mathrm{m}$ (includes mass of drilling mud and seawater);

$T=1.272191 \times 10^{6} \mathrm{~N}$ (tension at the bottom ball joint) $W=3123.095 \mathrm{~N} / \mathrm{m}$ (net weight of riser per unit length in seawater includes $554.79 \mathrm{~N} / \mathrm{m}$ for choke and kill lines); $\gamma_{w}=10179.3 \mathrm{~N} / \mathrm{m}^{3} ; \gamma_{f}=13352.4 \mathrm{~N} / \mathrm{m}^{3}$

Using MSTMM, the riser is discretized into 2 elements with equal length. Table 3 shows the first five natural frequencies in comparison with [24-26]. Good agreement has been achieved.

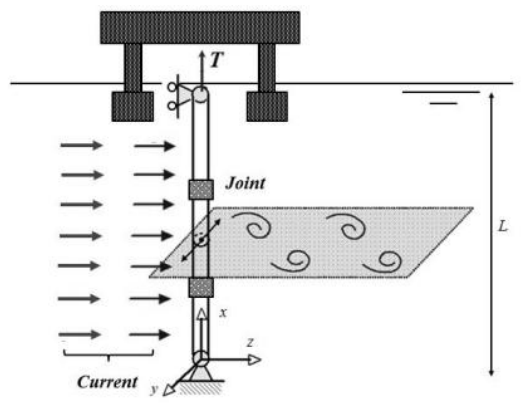

Figure 7. Sketch of the riser model

Sketch of composite riser and ocean platform under the action of current is shown in Fig. 7. The composite riser consists of RTP and steel joints. The length of the riser is $150 \mathrm{~m}$ with an outside diameter $0.365 \mathrm{~m}$ and for every $50 \mathrm{~m}$; one steel joint is used to connect the pipes. The bottom end of the riser is assumed to be hinged to the base, i.e., no translations and subject to the rotational constraint $\theta_{z}=$ free. At the top, the riser is assumed to be connected by a hinge to a floating structure, expressed as $x=$ free, $\theta_{z}=$ free with top tension $(T=60000 \mathrm{~N})$. Details of RTP material parameters are given in [27]. Following the procedures in Section $2.3 R_{e q}$ is obtained and equal to $8.7483 \times 10^{6} \mathrm{~N} . \mathrm{m}^{2}$. Total mass (RTP riser mass, oil mass and seawater added mass) is $198.7953 \mathrm{~kg} / \mathrm{m}$. Single element is used. Commercial software ANSYS Workbench using SHELL 181 element is involved in our analysis. 54500 elements and 54520 nodes are used to discretize the RTP riser. Finally, the analytical result of hinge-hinge pre-stress beam can be obtained from the following equation [28]:

$$
f_{n}(\mathrm{~Hz})=\left(\frac{n^{2} \pi}{2 L^{2}}\right) \sqrt{\frac{E I}{m}} \sqrt{1+\frac{T L^{2}}{n^{2} \pi^{2} E I}}, n=1,2,3, \mathrm{~L}
$$
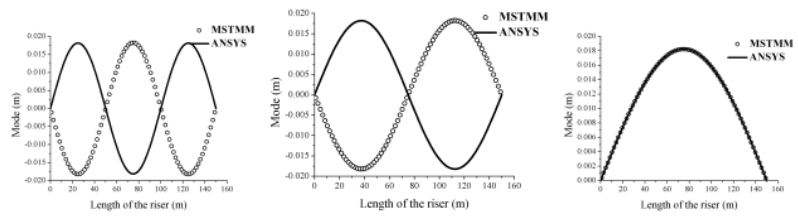

Figure 8. MSTMM and FEM ANSYS first three mode shapes results of RTP riser.

The computation results of the first three natural frequencies $(\mathrm{Hz})$ by MSTMM and FEM ANSYS are
$f_{1-3}=0.059732,0.057988[\mathrm{ANSYS}] ; 0.129791,0.12618$

[ANSYS]; 0.218070, 0.21232[ANSYS]. The first three mode shapes are demonstrated in Fig. 8. Good agreement has been achieved, which verified the accuracy of the MSTMM dynamic model.

\section{Conclusions}

Free vibration of flexible marine risers and pipelines on an elastic foundation conveying fluid has been extensively studied based on various available techniques with tedious calculations in many cases. An alternative procedure is proposed in this paper for determining the natural frequencies of Reinforced Thermoplastic Pipe (RTP) in the industry applications. The procedure uses the method of Transfer Matrix Method for Multibody Systems (MSTMM). It reduces the dynamics problem, precisely the Eigenvalue problem to an overall transfer equation, which only involves boundary state vectors. We always end up with a system of linear algebraic equations for the modal vibration coordinates of boundary states. Using numerical examples, the paper shows that the natural frequencies obtained by MSTMM agree very well with that published in the literature and produced by Workbench ANSYS.

\section{Acknowledgment}

The authors gratefully acknowledge the financial support of this research by Science Challenge Project (No. JCKY2016212A506-0104).

\section{References}

1. M. P. Païdoussis, Fluid Structure Interactions: Slender Structures and Axial Flow, New York: Academic Press (1998)

2. H. Ashley and G. Haviland, Bending vibration of pipe line containing flowing fluid. Journal of Applied Mechanics. 17 (3): 229-232 (1950)

3. M. Eisenberger and J. Clastornik, Vibrations and buckling of a beam on a variable Winkler elastic foundation. Journal of Sound and Vibration. 115 (2): 233-241 (1987)

4. Z. Ding, A general solution to vibrations of beams on variable Winkler elastic foundation. Computers and Structures. 47 (1): 83-90 (1993)

5. S. H. Farghaly and K. M. Zeid, An exact frequency equation for an axially loaded beam-mass-spring system resting on Winkler elastic foundation. Journal of Sound and Vibration. 185 (2): 357-363 (1995)

6. N. R. Naidu and G. V. Rao, Vibrations of initially stressed uniform beams on two-parameter elastic foundation. Computers and Structures. 57 (5): $941-$ 943 (1995)

7. M. A. De Rosa, Free vibrations of Timoshenko beams on two-parameter elastic foundation. Computers and Structures. 57 (1): 151-156 (1995)

8. Y. Ayvaz and K. Ozgan, Application of modified Vlasov model to free vibration analysis of beams 
resting on elastic foundations. Journal of Sound and Vibration. 255 (1): 111-127 (2002)

9. Y. Bai, W. Ruan, P. Cheng, B. Yu and W. Xu, Buckling of reinforced thermoplastic pipe (RTP) under combined bending and tension. Ships and Offshore Structures. 9 (5): 525-539 (2014)

10. C. J. Price, The "State of the Art" in composite material development and applications for the oil and gas industry. Proceedings of the Twelfth International Offshore and Polar Engineering Conference; Kitakyushu, Japan: 125-131 (26-31 May 2002)

11. D. G. Pavlou, Flow-induced instability of multilayered anisotropic pipelines. International Journal of Computational Methods and Experimental Measurements. 4 (4): 543-553 (2016)

12. X. T. Rui, L. Yun, Y. Lu, B. He and G. P. Wang, Transfer matrix method for multibody system and its application. Science Press, Beijing (2008) (In Chinese)

13. X. T. Rui, G. P. Wang, Y. Lu and L. Yun, Transfer matrix method for linear multibody system. Multibody System Dynamics. 19 (3): 179-207 (2008)

14. X. T. Rui, J. Zhang and Q. Zhou, Automatic deduction theorem of overall transfer equation of multibody system. Advances in Mechanical Engineering. 2014 (2): 1-12 (2014)

15. L. K. Abbas, Q. Zhou, D. Bestle and X. T. Rui, A unified approach for treating linear multibody systems involving flexible beams. Mechanism and Machine Theory. 107: 197-209 (2017)

16. D. Bestle, L. K. Abbas and X. T. Rui, Recursive eigenvalue search algorithm for transfer matrix method of linear flexible multibody systems. Multibody System Dynamics. 32 (4): 429-444 (2014)

17. G. L. Kuiper and A. V. Metrikine, On stability of a clamped-pinned pipe conveying fluid. Heron. 4 (3): 211-232 (2004)

18. G. Eslami, V. A. Maleki and M. Rezaee, Effect of open crack on vibration behavior of a fluid-conveying pipe embedded in a visco-elastic medium. Latin
American Journal of Solids and Structures. 13 (1): 136-154 (2016)

19. L. P. Kollár and G. S. Springer, Mechanics of composite structures. Cambridge University Press, Cambridge (2009)

20. C. Franciosi and A. Masi, Free vibrations of foundation beams on two-parameter elastic soil. Computers and Structures. 47 (3): 419-426 (1993)

21. M. A. De Rosa and M. J. Maurizi, The influence of concentrated masses and Pasternak soil on the free vibrations of Euler beams - exact solution. Journal of Sound and Vibration. 212 (4): 573-581 (1998)

22. W. Q. Chen, C. F. Lü, and Z. G. Bian, A mixed method for bending and free vibration of beams resting on a Pasternak elastic foundation. Applied Mathematical Modelling. 28 (10): 877-890 (2004)

23. K. R. Chellapilla and H. S. Simha, Vibrations of fluid-conveying pipes resting on two-parameter foundation. Open Acoustics Journal. 1 (1): 24-33 (2008)

24. D. W. Dareing and T. Huang, Natural frequencies of marine drilling risers. Journal of Petroleum Technology. 28 (7): 813-818 (1976)

25. J. R. Graves and D. W. Dareing, Direct method for determining natural frequencies of marine risers in deep water. Journal of Energy Resources Technology. 126 (1):47-53 (2004)

26. Y. Cheng, J. K. Vandiver and G. Moe, The linear vibration analysis of marine risers using the WKBbased dynamic stiffness method. Journal of Sound and Vibration. 251 (4): 750-760 (2002)

27. D. Y. Chen, L. K. Abbas, G. P. Wang, X. T. Rui and W. J. Lu, Numerical calculation of vortex-induced vibration of composite riser. Journal of Shanghai Jiaotong University. 51 (4): 495-503 (2017) (in Chinese)

28. B. Sanaati and N. Kato, Vortex-induced vibration (VIV) dynamics of a tensioned flexible cylinder subjected to uniform cross-flow. Journal of Marine Science and Technology. 18 (2): 247-261 (2013) 